\title{
Comparative evaluation of SARS-CoV-2 IgG assays against nucleocapsid and spike antigens
}

\author{
Mitra Rezaei $^{\mathrm{a}, \mathrm{b}}$, Mohammadhadi Sadeghi ${ }^{\mathrm{b}}$, Alireza Korourian ${ }^{\mathrm{c}}$, Payam Tabarsi $^{\mathrm{a}}$, Mihan Porabdollah ${ }^{\mathrm{d}}$, \\ Elham Askari ${ }^{\mathrm{d}}$, Esmaeil Mortaz ${ }^{\mathrm{a}}$, Shima Mahmoudie,*, Majid Marjani ${ }^{\mathrm{a}}$ and Ali Akbar Velayati ${ }^{\mathrm{a}}$ \\ ${ }^{a}$ Clinical Tuberculosis and Epidemiology Research Center, National Research Institute of Tuberculosis and Lung \\ Diseases, Shahid Beheshti University of Medical Sciences, Tehran, Iran \\ ${ }^{\mathrm{b}}$ Virology Research Center, National Research Institute of Tuberculosis and Lung diseases, Shahid Beheshti \\ University of Medical Sciences, Tehran, Iran \\ ${ }^{\mathrm{c}}$ Tehran Pathobiology Laboratory Center, Tehran, Iran \\ ${ }^{\mathrm{d}}$ Chronic Respiratory Disease Research Center, National Research Institute of Tuberculosis and Lung Disease, \\ Shahid Beheshti University of Medical Sciences, Tehran, Iran \\ ${ }^{\mathrm{e}}$ Pediatric Infectious Disease Research Center, Tehran University of Medical Sciences, Tehran, Iran
}

\begin{abstract}
.
BACKGROUND: There are few studies to compare antibody response against anti-spike (S) and anti- nucleoprotein (N) SARSCoV-2.

OBJECTIVE: The aim of this study was to evaluate the IgG antibody production against $\mathrm{S}$ and $\mathrm{N}$ antigens of the virus and their correlation with the time and severity of the disease.

METHODS: The IgG antibodies against $\mathrm{S}$ and $\mathrm{N}$ antigens of SARS-CoV-2 in serum specimens of 72 symptomatic patients who tested real-time reverse transcription polymerase chain reaction positive for SARS-CoV-2 were detected using the ELISA technique. Different antibody response was compared and the correlation with the time from disease onset and the severity was evaluated.

RESULTS: Forty-eight of $72(67 \%)$ patients tested positive for anti-SARS-CoV-2 antibodies, while 24 (33\%) did not have detectable antibodies. Comparison of antibody levels for $\mathrm{N}$ and $\mathrm{S}$ antibodies showed that they correlate with each other well $(r=$ $0.81 ; P<0.001)$. However, sensitivity of anti-S SARS-CoV-2 IgG and anti-N SARS-CoV-2 IgG was $30 \%$ and $60 \%$, during the first 7 days after symptom onset $(r=0.53 ; P=0.111)$, but increased to $73 \%$ and $68 \%$ at more than 1-week post symptom onset $(r=0.89, P=0.111)$, respectively. Cases with positive IgG response showed a decreased CD $8^{+} \mathrm{T}$ cells percentage compared to the negative IgG groups ( $26 \pm 14$ vs. $58 \pm 32, p=0.066$ in anti-N IgG group and $28 \pm 15$ vs. $60 \pm 45, p=0.004$ in anti-S IgG group, respectively).

CONCLUSION: Nearly one-third of the confirmed COVID-19 patients had negative serology results. Lower percent positivity at early time points after symptom onset (less than 1 week) was seen using anti-S SARS-COV-2 IgG kit compare to the anti-N SARS-CoV-2 IgG; therefore, clinicians should interpret negative serology results of especially anti-S SARS-CoV-2 IgG with caution.
\end{abstract}

Keywords: SARS-CoV-2, antibody response, spike, nucleoprotein, lymphocyte subsets

\footnotetext{
${ }^{*}$ Corresponding author: Shima Mahmoudi, Pediatric Infectious Disease Research Center, Tehran University of Medical Sciences, Children's Medical Center Hospital, Dr. Gharib Street, Keshavarz Boulevard, Tehran, Iran. Tel.: +98 216642 8996; Fax: +98 216642 8996; E-mail: sh-mahmoudi@sina.tums.ac.ir.
}

\section{Background}

SARS-CoV-2 and its related disease COVID-19 is associated with significant morbidity and mortality globally $[1,2]$. According to the latest report of World Health Organization (WHO), more than 60 million people be- 
ing infected, with 1,420,306 deaths as of November 27, 2020. Although the fatality rate of SARS-CoV-2 is lower than those of other coronaviruses that caused disasters in the past, its higher infectivity rate makes it worse [3], probably make it as one of the biggest health and economic burden of the last 100 years [4].

Since there are no specific therapeutic drugs or vaccines for COVID-19, early detection of cases with SARS-CoV-2 infection is crucial to decrease the risk of infecting a larger population [5]. There are a number of important unanswered questions yet. First, it is uncertain how long antibodies persist after infection [6]. Second, SARS-CoV-2 serologic test could really be used in the clinical practice or not [7]; and the third, there are currently no studies which demonstrated that antibodies are protective against reinfection in humans [8,9].

Serological tests typically detect antibodies against spike protein $(\mathrm{S})$ and/or nucleoprotein $(\mathrm{N})$, the most immunogenic proteins of SARS-CoV-2. The S protein, consisting of a $\mathrm{S} 2$ and a $\mathrm{S} 1$ subunit is present on the envelope of SARS-CoV-2 and help the virus to connect to the human cells using the Angiotensin-converting enzyme 2 (ACE2) receptor [8]. Since anti-S protein antibodies have been shown to possess neutralizing effects in vitro, it has been suggested that detection of antibodies against $S$ protein could provide a better indication of an effective immune response [10,11].

There are few studies to evaluate the SARS-CoV-2 IgG assays, and lymphocyte subsets comprehensively in COVID-19 patients with different disease severity [12], and antibody response against anti-S and anti-N SARSCoV-2.

The aim of this study was to determine the antibody response against SARS-CoV-2 $\mathrm{S}$ and $\mathrm{N}$ protein using ELISAs for the detection of IgG and the presumptive correlation with level of lymphocyte subsets in COVID19 patients

\section{Methods}

This study was performed at the Masih Daneshvari Hospital, Tehran, Iran and approved by the local ethics committee (approval number: IR.SBMU.MSP.REC. 1399.260).

Seventy-two symptomatic patients who tested realtime reverse transcription polymerase chain reaction (RT -PCR) was positive for SARS-CoV-2 in nasopharyngeal swab samples and admitted to the infectious disease ward were recruited into the study. The presence of SARS-CoV-2 was detected as previously de- scribed [5]. Demographic data, laboratory parameters, and clinical severity during the hospitalization period were retrieved from patient records. The COVID-19 patients were classified into moderate, severe, and critical groups [12]. For the purpose of this study, at rest oxygen saturation $\left(\mathrm{O}_{2}\right.$ sat) and respiratory rate were used for severity classification. Patients with pulmonary infiltration in chest imaging and $\mathrm{O}_{2}$ sat more than $93 \%$ with ambient air were classified as moderate group and patients with $\mathrm{O}_{2}$ sat $\leqslant 93 \%$ or a respiratory rate of more than 30 breaths/min were categorized as severe group. The patients, who need noninvasive or mechanical ventilation; and the patients with shock, or who need intensive care management, were classified as critical cases.

Days of symptoms were recorded based on first day of onset of COVID-19 symptoms, as documented by managing clinicians. In addition, we collected COVID19 patients who have detected lymphocyte subsets and SARS-CoV-2 antibodies during the same day.

\subsection{SARS-CoV-2 antibody detection}

For every patient, one blood sample was collected. The serum IgG antibodies against $\mathrm{N}$ and $\mathrm{S}$ antigens of SARS-CoV-2 were measured according to the manufacturers' instructions using the enzyme-linked immunosorbent assay (ELISA) kits supplied by Pishtaz Teb Diagnostics Company, and EUROIMMUN antiSARS-CoV-2 assay kits.

\subsection{Flow cytometry analysis}

The percentages and absolute counts of total $\mathrm{T}$ cells, $\mathrm{CD}^{+}{ }^{+} \mathrm{T}$ cells, $\mathrm{CD}^{+} \mathrm{T}$ cells, B cells, and NK cells were determined by using phycoerythrin conjugated anti-human CD4, CD19, CD56 antibodies; anti-human CD8 and CD16 allophycocyanin conjugated antibodies; and fluorescein sothiocyanate conjugated antibody for $\mathrm{CD}^{+} \mathrm{T}$ cells according to the manufacturer's (PharMingen) instructions. A FACSCalibur ${ }^{\mathrm{TM}}$ flow cytometer (Becton Dickinson, San Jose, CA, USA) was used for cell analysis.

\subsection{Statistical analysis}

Statistical analysis was performed using SPSS 16.0 software. Measurement data were tested for normality. Data that confirmed normality were expressed as mean \pm standard deviation $(S D)$, and $t$-test was used for comparison between groups. Median and interquartile range 
(IQR) were used for noncompliant data. The comparison between multiple groups, Kruskal-Wallis test was used for pairwise comparison between groups. Pearson correlation tests were also performed. A two-sided $P$ value of less than 0.05 was considered statistically significant.

\section{Results}

A total of 72 patients with COVID-19 admitted to the Masih Daneshvari Hospital, Tehran, Iran were enrolled in the study. The majority of the patients with RT-PCRconfirmed SARS-CoV-2 were female $(57 \%, n=41)$, and the median age was 60 years (IQR: $45-68$ years). Forty-eight $(67 \%)$ of the patients had $\geqslant 1$ risk factor, including heart disease, chronic lung disease, diabetes mellitus, and hypertension. The cases were classified into three groups, moderate (11 cases, $15 \%)$, severe (27 cases, 38\%), and critical (34 cases, $47 \%$ ).

A total of 72 plasma samples were collected during the hospitalization and tested for antibodies against SARS-CoV-2 S and N antigens. Forty-eight of 72 (67\%) patients tested positive for anti-SARS-CoV-2 Abs with either Pishtaz Teb or EUROIMMUN anti-SARS-CoV-2 Assay, while 24 (33\%) did not have detectable antibodies. Although the number of serology positive cases using Pishtaz Teb or EUROIMMUN anti-SARS-CoV-2 kits was similar, 3 cases had positive anti-N SARSCoV-2 IgG and negative anti-S SARS-CoV-2 IgG tests. On the other hand, 3 cases showed detectable antiS SARS-CoV-2 IgG tests, while the negative anti-N SARS-CoV-2 IgG was found.

The mean duration from onset of symptoms to perform anti-N and anti-S IgG test was close between negative and positive anti-N IgG groups (17.0 \pm 8.8 vs. $17.31 \pm 10.7$ days) and negative and positive anti-S IgG groups $(15.7 \pm 9.4$ vs. $17.7 \pm 10.4$ days $)$, respectively.

In the current study, sensitivity of anti-S SARS-CoV$2 \mathrm{IgG}$ and anti-N SARS-CoV-2 IgG was $30 \%$ and $60 \%$, respectively during the first 7 days after symptom onset, but increased to $73 \%$ and $68 \%$ at more than 1-week post symptom onset (Table 1).

The median level of anti-S SARS-CoV-2 IgG during the first week after onset of symptoms was 0.45 (IQR: 0.29-2.9) that was significantly lower than the observed anti-S SARS-CoV-2 IgG in groups who were sampled after 1 week from onset of symptoms (9.0; IQR: 0.6$13.6, p$ value $=0.004)$; while no significant difference was found in the level of anti-N SARS-CoV-2 IgG in groups during the first week and after 1 week from onset of symptoms (10. (IQR: 0.2-20.8) vs. 14.2 (IQR: $0.4-25.6) ; p$ value $=0.34)$.

The median level of both anti-S SARS-CoV-2 IgG and anti-N SARS-CoV-2 IgG in severe and critical illness patients were not differ significantly compare to those in moderate course of disease $(p=0.46$ and $p=$ 0.21 , respectively).

Comparison of antibody levels for $\mathrm{N}$ and $\mathrm{S}$ antibodies showed that they correlate with each other well $(r=$ $0.81 ; P<0.001)$. Among the RT-PCR-positive patient samples collected $>14$ days after onset of symptoms, seropositive $\mathrm{N}$ antibodies were detected in 24 out of 34 samples, yielding a sensitivity of $63 \%$. A similar analysis of the spike antibody in samples collected $>14$ days after onset of symptoms showed a slightly higher sensitivity of $66 \%$ (25 of 38) ( $r=0.94 ; P<0.001)$, while their correlation among samples collected $<14$ days after onset of symptoms was lower $(r=0.66 ; P<$ 0.001).

Lower percent positivity at early time points after symptom onset (less than 1 week) was seen using anti-S SARS-COV-2 IgG kit compare to the anti-N SARSCoV-2 IgG $(r=0.53 ; P=0.111)$, while anti-S SARSCOV-2 IgG in samples collected $>7$ days after onset of symptoms showed a slightly higher sensitivity compare to the anti-N SARS-COV-2 IgG kit (73\% versus $68 \%$, respectively; $r=0.89, P=0.111$ ).

Further, compared to the negative anti-N and anti-S IgG group, the neutrophil counts were lower in the anti$\mathrm{N}$ IgG positive group $(6.3 \pm 1.0$ vs. $19.9 \pm 9.7, p<$ $0.001)$ and anti-S IgG positive group (6.4 \pm 1.61 vs. 9.4 $\pm 6.01, p=0.005)$, respectively; while the counts of total WBC and lymphocyte were not significantly differ in negative and positive anti-N or anti-S IgG groups.

The antibody levels and lymphocyte subsets of 14 COVID-19 patients were evaluated and cases with positive IgG response showed a decreased CD8 cell percentage compared to the negative IgG groups $(26 \pm 14$ vs. $58 \pm 32, p=0.066$ in anti-N IgG group and $28 \pm$ 15 vs. $60 \pm 45, p=0.004$ in anti-S IgG group, respectively). No significant differences were found between antibody levels and other lymphocyte subsets.

\section{Discussion}

Our data showed that for both $\mathrm{N}$ and $\mathrm{S}$ antigens, the sensitivity was $67 \%$, and $33 \%$ did not have detectable antibodies, so negative serological results alone cannot exclude the diagnosis of COVID-19 that is consistent with the previous report [13]. Comparison of antibody 
Table 1

The sensitivity of anti-N SARS-COV-2 IgG and anti-N SARS-COV-2 IgG assays during the time

\begin{tabular}{|c|c|c|c|c|c|c|c|c|c|c|c|}
\hline & & \multicolumn{2}{|c|}{$\begin{array}{l}\text { Less than } \\
1 \text { week }\end{array}$} & \multicolumn{2}{|c|}{$\begin{array}{c}\text { More than } \\
1 \text { week }\end{array}$} & \multirow[t]{2}{*}{$P$ value } & \multicolumn{2}{|c|}{$\begin{array}{c}\text { Less than } \\
2 \text { weeks }\end{array}$} & \multicolumn{2}{|c|}{$\begin{array}{l}\text { More than } \\
2 \text { weeks }\end{array}$} & \multirow[t]{2}{*}{$P$ value } \\
\hline & & $\mathrm{N}$ & $\%$ & $\mathrm{~N}$ & $\%$ & & $\mathrm{~N}$ & $\%$ & $\mathrm{~N}$ & $\%$ & \\
\hline \multirow[t]{2}{*}{ Anti-N SARS-COV-2 IgG } & Negative & 4 & 40 & 20 & 32 & 0.720 & 10 & 29 & 14 & 37 & 0.62 \\
\hline & Positive & 6 & 60 & 42 & 68 & & 24 & 71 & 24 & 63 & \\
\hline \multirow[t]{2}{*}{ Anti-S SARS-COV-2 IgG } & Negative & 7 & 70 & 17 & 27 & 0.013 & 11 & 32 & 13 & 34 & 1.0 \\
\hline & Positive & 3 & 30 & 45 & 73 & & 23 & 68 & 25 & 66 & \\
\hline
\end{tabular}

levels for $\mathrm{N}$ and $\mathrm{S}$ antigens showed that they correlate with each other well $(r=0.81 ; P<0.001)$. The sensitivity for antibody to the $\mathrm{N}$ protein for samples collected $\leqslant 7$ days after onset of symptoms was $60 \%$ (6 of 10). Analysis of $\mathrm{S}$ antibodies at this time point showed a reduced sensitivity of $30 \%$ ( 3 of 10). Taken together, timing of when the tests are used is important [14] and our findings indicate that detection of antibodies against the $\mathrm{N}$ protein is more sensitive than detection of antibodies against the $\mathrm{S}$ protein during the first week after symptom onset, and that $\mathrm{N}$ antibodies generally appear earlier than spike antibodies that is in consistent with previous report [15]. At the onset of SARS-CoV infection, B cells elicit an early response against the $\mathrm{N}$ protein, while antibodies against $\mathrm{S}$ protein could be detected after $4-8$ days from the early stage of acute infection $[3,16]$. N protein is an internal viral protein of SARS-CoV-2 and is not a target of neutralizing antibodies, so earlier and even stronger anti-N antibody production might observe [17].

According to the previous report, although nearly 93\% of exposed asymptomatic individuals had detectable $\mathrm{T}$ cell responses to SARS-CoV-2, only $60 \%$ of cases were seropositive [18]. In the current study, we found that $36 \%(n=20)$ and $34 \%(n=19)$ of the recovered patients had negative anti- $\mathrm{N}$ and anti$\mathrm{S}$ IgG, respectively. The definite mechanism remains unclear [19].

Several studies reported that weak or non-responders for IgG antibody had higher viral clearance than strong responders and robust antibody response correlate with the severity of the disease $[6,20]$, while in our study similar to previous reports $[6,21]$, antibody response in severe and critical illness patients were not differ significantly compare to those in moderate course of disease. We concluded that antibody levels could not be used to predict the severity of the disease that was in consistent with previous reports.

In our study, the neutrophil counts were lower in the IgG positive group compared to the negative $\mathrm{IgG}$ group that is consistent with Liu et al. study [19]. Cases with positive $\mathrm{IgG}$ response showed a decreased CD8 cell percentage compared to the negative $\operatorname{IgG}$ groups ( 26 \pm 14 vs. $58 \pm 32, p=0.066$ in anti-N IgG group and $28 \pm 15$ vs. $60 \pm 45, p=0.004$ in anti-S IgG group, respectively), while no significant differences was observed between antibody levels and the counts of other lymphocyte subsets in COVID-19 patients, which might be due to that the detection of lymphocyte subsets could not reflect the specific $\mathrm{T}$ cell or plasma cell levels during SARS-CoV-2 infection [12]. Our results are consistent with Zhang et al. that reported no association between antibody levels and the $\mathrm{T}$ cells, $\mathrm{CD} 4^{+} \mathrm{T}$ cells, $\mathrm{CD} 8^{+} \mathrm{T}$ cells, NK cells, and B cells [22].

The strength of our study includes the using the same cohort of unique, non-duplicate COVID-19 patients' sera to compare performance of anti-S and antiN SARS-CoV-2 IgG response head-to-head. There are a number of limitations to our study. First, we only included a limited number of samples particularly for determination of lymphocyte subsets. Second, the control samples were not included for calculation of specificity. Third, we did not follow the patients for evaluating possible seroconversion. Finally, we only evaluated the diagnostic performance in patients with moderate to critical COVID-19 and did not study the antibody response in asymptomatic persons and patients with mild COVID-19.

\section{Conclusion}

In our study, nearly one-third of the confirmed COVID-19 patients had negative serology results. Compared to the anti-N SARS-CoV-2 IgG assay, anti-S SARS-COV-2 assay showed lower sensitivity during the first week after symptom onset; therefore, clinicians should interpret negative serology results of especially anti-S SARS-CoV-2 IgG with caution. Further investigation of patients who fail to produce detectable levels of IgG is highly recommended.

\section{Acknowledgments}

This study was taken from Dr. Mohammadhadi Sadeghi's postgraduate thesis. 


\section{Conflict of interest}

The authors declare that they have no competing interests.

\section{References}

[1] S. Mahmoudi, M. Rezaei, N. Mansouri, M. Marjani and D. Mansouri, Immunologic features in coronavirus disease 2019: functional exhaustion of T cells and cytokine storm, $J$ Clin Immunol 40 (2020), 974-976.

[2] M. Ekbatani, S. Hassani, L. Tahernia, B. Yaghmaei, S. Mahmoudi, A. Navaeian, M. Rostamyan, F. Zamani and S. Mamishi, Atypical and novel presentations of coronavirus disease 2019: A case series of three children, Br J Biomed Sci 78(1) (2021), 47-52.

[3] V.K. Shah, P. Firmal, A. Alam, D. Ganguly and S. Chattopadhyay, Overview of immune response during SARS-CoV-2 infection: Lessons from the past, Front Immunol 11 (2020), 1949.

[4] A.K. Azkur, M. Akdis, D. Azkur, M. Sokolowska, W. van de Veen, M.C. Brüggen, L. O’Mahony, Y. Gao, K. Nadeau and C.A. Akdis, Immune response to SARS-CoV-2 and mechanisms of immunopathological changes in COVID-19, Allergy 75 (2020), 1564-1581.

[5] S. Mahmoudi, M. Mehdizadeh, R.S. Badv, A. Navaeian, B. Pourakbari, M. Rostamyan, M.S. Ekbatani, H. Eshaghi, M.R. Abdolsalehi and H. Alimadadi, The coronavirus disease 2019 (COVID-19) in children: A study in an Iranian Children's Referral Hospital, Infect Drug Resist 13 (2020), 2649-265.

[6] Q.-X. Long, X.-J. Tang, Q.-L. Shi, Q. Li, H.-J. Deng, J. Yuan, J.-L. Hu, W. Xu, Y. Zhang and F.-J. Lv, Clinical and immunological assessment of asymptomatic SARS-CoV-2 infections, Nat Med 26 (2020), 1200-1204.

[7] E.S. Theel, P. Slev, S. Wheeler, M.R. Couturier, S.J. Wong and K. Kadkhoda, The role of antibody testing for SARS-CoV-2: is there one? J Clin Microbiol 58(8) (2020), e00797-20.

[8] J. Van Elslande, B. Decru, S. Jonckheere, E. Van Wijngaerden, E. Houben, P. Vandecandelaere, C. Indevuyst, M. Depypere, S. Desmet and E. André, Antibody response against SARS-CoV2 spike protein and nucleoprotein evaluated by four automated immunoassays and three ELISAs, Clin Microbiol Infect 26(11) (2020), 1557e1-1557e7.

[9] M.K. Özçürümez, A. Ambrosch, O. Frey, V. Haselmann, S. Holdenrieder, M. Kiehntopf, M. Neumaier, M. Walter, F. Wenzel and R. Wölfel, SARS-CoV-2 Antibody Testing - Questions to be asked, J Allergy Clin Immunol 146(1) (2020), 35-43.

[10] F. Amanat, D. Stadlbauer, S. Strohmeier, T.H. Nguyen, V. Chromikova, M. McMahon, K. Jiang, G.A. Arunkumar, D. Jurczyszak and J. Polanco, A serological assay to detect SARSCoV-2 seroconversion in humans, Nat Med 26(7) (2020), 1033-1036.
[11] R.A. Perera, C.K. Mok, O.T. Tsang, H. Lv, R.L. Ko, N.C. Wu, M. Yuan, W.S. Leung, J.M. Chan and T.S. Chik, Serological assays for severe acute respiratory syndrome coronavirus 2 (SARS-CoV-2), March 2020, Euro Surveill 25 (2020), 2000421.

[12] D. Sun, H. Li, X.-X. Lu, H. Xiao, J. Ren, F.-R. Zhang and Z.-S. Liu, Clinical features of severe pediatric patients with coronavirus disease 2019 in Wuhan: a single center's observational study, World J Pediatr 16(3) (2020), 251-259.

[13] S. Zheng, J. Fan, F. Yu, B. Feng, B. Lou, Q. Zou, G. Xie, S. Lin, R. Wang and X. Yang, Viral load dynamics and disease severity in patients infected with SARS-CoV-2 in Zhejiang province, China, January-March 2020: Retrospective cohort study, BMJ 369 (2020), m1443.

[14] J.J. Deeks, J. Dinnes, Y. Takwoingi, C. Davenport, R. Spijker, S. Taylor-Phillips, A. Adriano, S. Beese, J. Dretzke and L.F. di Ruffano, Antibody tests for identification of current and past infection with SARS-CoV-2, Cochrane Database Syst Rev 6(6) (2020), CD013652.

[15] P.D. Burbelo, F.X. Riedo, C. Morishima, S. Rawlings, D. Smith, S. Das, J.R. Strich, D.S. Chertow, R.T. Davey Jr, and J.I. Cohen, Sensitivity in detection of antibodies to nucleocapsid and spike proteins of severe acute respiratory syndrome coronavirus 2 in patients with coronavirus disease 2019, J Infect Dis 222(2) (2020), 206-213.

[16] Y.-J. Tan, P.-Y. Goh, B.C. Fielding, S. Shen, C.-F. Chou, J.-L. Fu, H.N. Leong, Y.S. Leo, E.E. Ooi and A.E. Ling, Profiles of antibody responses against severe acute respiratory syndrome coronavirus recombinant proteins and their potential use as diagnostic markers, Clin Diagn Lab Immunol 11 (2004), 362 371.

[17] D.T.M. Leung, T.F. Chi Hang, M. Chun Hung, P.K. Sheung Chan, J.L.K. Cheung, H. Niu, J.S.L. Tam and P.L. Lim, Antibody response of patients with severe acute respiratory syndrome (SARS) targets the viral nucleocapsid, J Infect Dis 190 (2004), 379-386.

[18] P.F. Cañete and C.G. Vinuesa, COVID-19 makes B cells forget, but T cells remember, Cell 183 (2020), 13-15.

[19] J. Liu, J. Guo, Q. Xu, G. Cai, D. Chen and Y. Shen, Detection of IgG antibody during the follow-up in patients with COVID19 infection, Crit Care 24(1) (2020), 448.

[20] L. Lan, D. Xu, G. Ye, C. Xia, S. Wang, Y. Li and H. Xu, Positive RT-PCR test results in patients recovered from COVID-19, JAMA 323 (2020), 1502-1503.

[21] W.S. Phipps, J.A. SoRelle, Q.-Z. Li, L. Mahimainathan, E. Araj, J. Markantonis, C. Lacelle, J. Balani, H. Parikh and E.B. Solow, SARS-CoV-2 Antibody responses do not predict COVID-19 disease severity, Am J Clin Pathol 154 (2020), 459-465.

[22] B. Zhang, D. Yue, Y. Wang, F. Wang, S. Wu and H. Hou, The dynamics of immune response in COVID-19 patients with different illness severity, J Med Virol 93(2) (2021), 1070-1077. 